\title{
Coxsackievirus B3-Associated Myocardial Pathology and Viral Load Reduced by Recombinant Soluble Human Decay-Accelerating Factor in Mice
}

\author{
Bobby Yanagawa, O. Brad Spiller, Jonathan Choy, Honglin Luo, Paul Cheung, \\ Huifang M. Zhang, lan G. Goodfellow, David J. Evans, Agripina Suarez, \\ Decheng Yang, and Bruce M. McManus
}

UBC McDonald Research Laboratories/The iCAPTUR ${ }^{4} E$ Centre (BY, JC, HL, PC, HMZ, AS, DY), Department of Pathology and Laboratory Medicine, St. Paul's Hospital/Providence Health Care-University of British Columbia, Vancouver British Columbia, Canada; and Complement Biology and Virus Immune Evasion Group (OBS), University of Wales College of Medicine, Department of Medical Biochemistry, Cardiff, and Division of Virology (IGG, DJE), Institute of Biomedical and Life Sciences, University of Glasgow, Glasgow, Scotland, United Kingdom

\begin{abstract}
SUMMARY: Coxsackievirus B3 (CVB3) infection can result in myocarditis, which in turn may lead to a protracted immune response and subsequent dilated cardiomyopathy. Human decay-accelerating factor (DAF), a binding receptor for CVB3, was synthesized as a soluble IgG1-Fc fusion protein (DAF-Fc). In vitro, DAF-Fc was able to inhibit complement activity and block infection by CVB3, although blockade of infection varied widely among strains of CVB3. To determine the effects of DAF-Fc in vivo, 40 adolescent $\mathrm{A} / \mathrm{J}$ mice were infected with a myopathic strain of CVB3 and given DAF-Fc treatment 3 days before infection, during infection, or 3 days after infection; the mice were compared with virus alone and sham-infected animals. Sections of heart, spleen, kidney, pancreas, and liver were stained with hematoxylin and eosin and submitted to in situ hybridization for both positive-strand and negative-strand viral RNA to determine the extent of myocarditis and viral infection, respectively. Salient histopathologic features, including myocardial lesion area, cell death, calcification and inflammatory cell infiltration, pancreatitis, and hepatitis were scored without knowledge of the experimental groups. DAF-Fc treatment of mice either preceding or concurrent with CVB3 infection resulted in a significant decrease in myocardial lesion area and cell death and a reduction in the presence of viral RNA. All DAF-Fc treatment groups had reduced infectious CVB3 recoverable from the heart after infection. DAF-Fc may be a novel therapeutic agent for active myocarditis and acute dilated cardiomyopathy if given early in the infectious period, although more studies are needed to determine its mechanism and efficacy. (Lab Invest 2003, 83:75-85).
\end{abstract}

C oxsackievirus B3 (CVB3), an enterovirus of the family Picornaviridae, is a major cause of viral myocarditis (Woodruff, 1980). Virus infection typically requires the presence of a specific and viable receptor, permissiveness of the host cell to viral replication, and a favorable virus-host interaction to ensure viability of viral progeny (Kunin, 1964; Shafren et al, 1995). Coxsackieviruses recognize the coxsackievirus and adenovirus receptor (CAR) and the decay-accelerating factor (DAF) coreceptor proteins to facilitate infection.

\section{DOI: 10.1097/01.LAB.0000049349.56211.09}

Received September 26, 2002.

Supported by Canadian Institutes of Health Research (grant-in-aid to $B M M$, studentships to BY and JC), Heart and Stroke Foundation of Canada (studentships to BY and JC), Heart and Stroke Foundation of British Columbia and Yukon (grant-in-aid to BMM), Michael Smith Foundation for Health Research (studentships to BY and JC), and Wellcome Trust Career Development Fellowship (Grant 061150/Z/00/Z to $O B S$ ).

Address reprint requests to: Dr. B. McManus, Cardiovascular Research Laboratory, UBC-St. Paul's Hospital, 1081 Burrard Street, Vancouver B.C., Canada V6Z 1Y6.E-mail: bmcmanus@mrl.ubc.ca
CAR is a 46-kDa protein, identified as a CVB3 receptor that mediates attachment and infection by CVB and adenovirus 2 and 5 (Bergelson et al, 1998; Krah and Crowell, 1982). As a member of the immunoglobulin superfamily, CAR has been shown to interact with the immune system through immunoglobulin binding (Carson and Chapman, 2001). Recently, CAR has been shown to interact with ZO-1 and has been implicated as a transmembrane protein of the epithelial tight junctional complex (Cohen et al, 2001). DAF, also known as CD55, is made up of four short consensus repeat (SCR) sequences, an $\mathrm{S} / \mathrm{T}$ region, and a glycosylphosphatidylinositol anchor to the outer leaflet of the cell membrane (Nicholson-Weller and Wang, 1994). CVB3 binding to DAF has been mapped to SCR 2-3 (Bergelson et al, 1995), and DAF binding has been shown to trigger tyrosine kinase-dependent cell signaling, previously found to be necessary for infection (Liu et al, 2000). The DAF receptor may facilitate initial virus attachment and CAR-binding efficiency to the canyon of the virus protein coat (Rossmann et al, 1985; Shieh and Bergelson, 2002). As a membranebound protein member of the family of regulators of 
complement activation, DAF affords resistance to complement-mediated attack and subsequent host cell damage. This inhibitory mechanism occurs by dissociation of C3 and C5 convertases, which blocks both classical and alternative complement pathways (Nicholson-Weller and Wang, 1994; Nicholson-Weller et al, 1982). It is tempting to speculate that CVB3 evolved to use a cellular receptor and coreceptor to modify the immune response to enhance viral spread (Ito et al, 2000). The relationship between cellular receptors and viral heart disease is particularly important as reflected in the strong correlation between increased receptor expression in neonatal hearts and increased susceptibility to virus infection (Liu and Opavsky, 2000).

Current management of active myocarditis is based on supportive therapy for systolic dysfunction (Batra and Lewis, 2001). Several attempts have been made to block myocarditis pathogenesis by intersecting various biological checkpoints, such as by immune suppression with a combination of prednisone and cyclosporine or azathioprine (Mason et al, 1995) or by immune enhancement with IFN- $\alpha$ for enteroviral myocarditis (Maisch et al, 1995). Work by our group and others has revealed the importance of direct virus infection, as opposed to immune injury, as a major contributor to early myocardial damage (Carthy et al, 1998; Chow et al, 1992; Liu and Opavsky, 2000). These insights have inspired therapies that directly target the virus itself. To this point, pleconaril, a picornavirus protein-coat binding agent, is one such therapy that has been administered to successfully attenuate acute enterovirus infection (Bauer et al, 2002; Rotbart and Webster, 2001). Other therapeutic approaches that target the enteroviruses, including coxsackieviruses, include antisense oligonucleotide therapy (Wang et al, 2001), mAbs to CAR (Martino et al, 2000), and WIN compound antiviral agents (Fohlman et al, 1993). Such therapies may be efficacious in the treatment of fulminant and potentially lifethreatening infections for which an enteroviral etiology can be readily identified.

Our laboratory has previously shown that the complement protein C3 interacts with CVB3 capsid proteins and is necessary for viral antigen retention in splenic germinal centers (Anderson et al, 1996). Such interactions activate the alternative pathway of complement and are important for germinal center formation. Previous attempts to attenuate infection with the use of anti-DAF antibodies have been successful in blocking virus infection of susceptible cells in vitro, demonstrating the importance of virus receptor binding to the development of infection (Martino et al, 1998; Shafren et al, 1997). Here, we have expressed and purified a novel soluble, dimeric DAF receptor ligated to human IgG1-Fc (DAF-Fc) as a potential inhibitor of CVB3 infection. We set out to determine whether such treatment can attenuate virus infection both in vitro and in vivo.

\section{Results}

\section{Synthesis of DAF-FC}

The cDNA encoding the four SCR of human DAF was cloned into an expression vector in frame and upstream from cDNA encoding the carboxy terminus of the human IgG1 $\mathrm{Fc}$ region (Harris et al, 2000). The expression vector was stably transfected into Chinese hamster ovary cells, and the supernatant of these cells contained the DAF-Fc fusion protein. The Fc region was added to HDAF for three reasons: (1) It enhanced the secretion of the soluble protein into the supernatant of transfected cells. (2) It greatly increased the ease of purifying the resultant soluble protein, using a protein A-coupled Sepharose column. (3) It enhanced the stability of the soluble protein in vivo (Harris et al, 2002). Because of the nature of the Fc portion of the fusion protein, the recombinant protein is dimeric, resembling an IgG molecule with the Fab regions exchanged for the inserted protein, and with a resultant $M_{r}=150 \mathrm{kDa}$ (Fig. 1). The purity of protein A-Sepharose-purified DAF-Fc was confirmed by silver stained SDS-PAGE and the concentration determined by sandwich ELISA. As a control for the non-DAF portion of the fusion protein, the Fc portion was released from DAF-Fc by incubation with agaroseimmobilized papain and the cleaved Fc tail purified from the released DAF by protein A-Sepharose capture and elution (data not shown). Free Fc was buffer exchanged to PBS and condensed to the same concentration as uncleaved DAF-Fc for in vivo experiments.

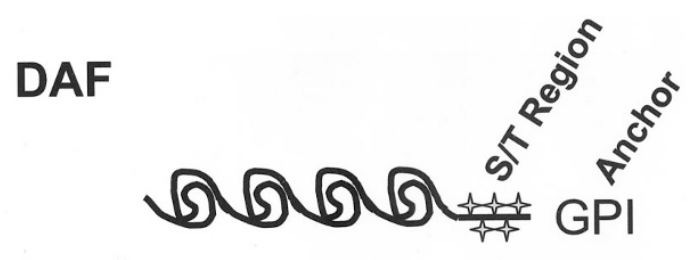

\section{DAF-Fc}

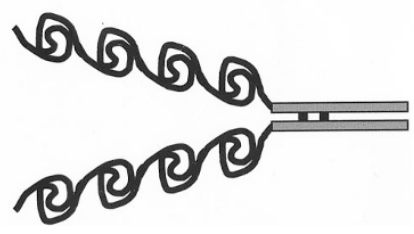

Figure 1.

Human decay-accelerating factor (HDAF) and the DAF-Fc molecules. DAF is made up of the four short consensus repeat (SCR) sequences, an S/T region, and a glycosylphosphatidylinositol (GP) anchor. The dimeric DAF-Fc molecule contains two to four SRC sequence regions ligated to the hinge and Fc portion of a human $\lg G$ antibody. 


\section{Complement Regulation and Virus Blockage by DAF-Fc In Vitro}

The ability of DAF-Fc to inhibit complement-mediated lysis of antibody-sensitized sheep erythrocytes was measured after incubation with 10\% normal human serum (Fig. 2). The inhibition by DAF-Fc was compared with DAF that had been released in a monomeric state by papain-cleavage of the Fc fusion protein. Both dimeric DAF-Fc and the released monomeric DAF were able to inhibit complementmediated lysis (Fig. 2). More DAF-Fc was required to reach the same level of complement inhibition as monomeric DAF, but comparing the efficacy of these molecules is difficult because DAF-Fc is composed of two DAF moieties per molecule and the Fc portion contributes to the overall molecular mass. However, both DAF-Fc and monomeric DAF were similar in their ability to regulate complement-mediated lysis, indicating that there was little steric or conformational impediment to the function of DAF when present as an immunoglobulin-like dimer. We then tested the ability of DAF-Fc to block CVB infection of IB-RS-2 cells (Fig. 3), which lack DAF and are infected by CVB in a CAR-dependent manner, as would occur in the in vivo mouse model (Spiller et al, 2000, 2002). Figure 3 shows that preincubation of three different strains of CVB3 with $75 \mu \mathrm{g} / \mathrm{ml}$ DAF-Fc resulted in different degrees of infection inhibition. A 10-fold decrease in infectivity was observed for the Gauntt strain, and no effect was observed for the Kandolf strain, both of which are established cardiovirulent strains. However, DAF-Fc was able to reduce the infectivity of the CVB-RD strain by greater than $10^{4}$-fold.

\section{Assessing the Efficacy of DAF-Fc In Vivo}

Myocarditis-susceptible A/J mice were infected intraperitoneally with $10^{5}$ plaque-forming units (PFU) of CVB3 (Gauntt) and treated intravenously with DAF-Fc 3 days before infection (preDAF), concurrent with

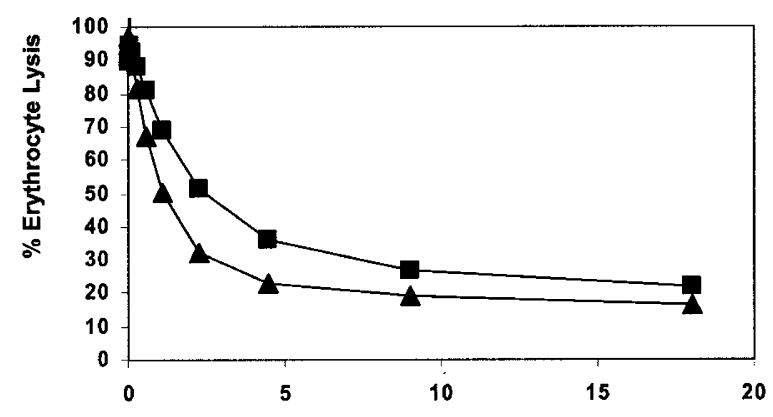

Final Concentration of DAF $(\mu \mathrm{g} / \mathrm{ml})$

Figure 2.

Complement inhibition of erythrocyte lysis by HDAF. Antibody-sensitized sheep erythrocytes were incubated with $10 \%$ normal human serum in the presence of dilutions of dimeric DAF-Fc (square) or monomeric DAF (triangle). Released hemoglobin was measured at $415 \mathrm{~nm}$, and the percent of total lysis was calculated compared with total lysis observed after distilled water addition to erythrocytes. Complement-mediated lysis was inhibited by $50 \%$ when approximately 2 to $3 \mu \mathrm{g} / \mathrm{ml}$ of monomeric DAF or DAF-Fc was added.
Virus Dilution:

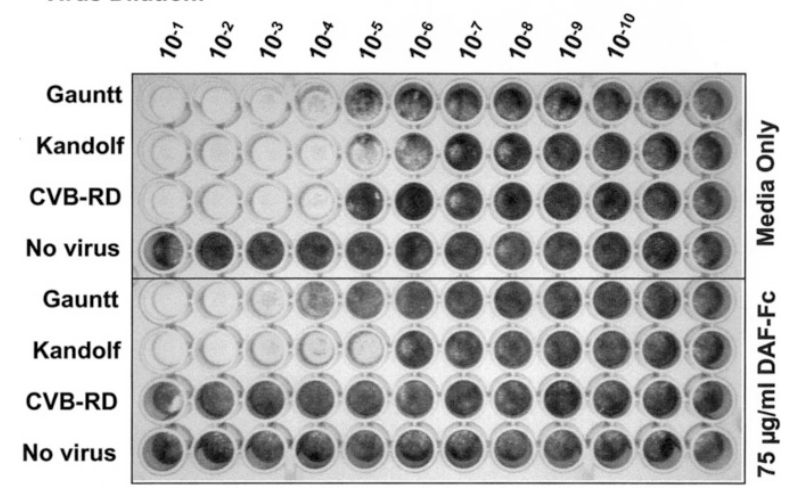

Figure 3.

DAF-Fc inhibition of Coxsackievirus B3 (CVB3)-induced cytopathic effect in IB-RS-2 cells. Preincubation of Gauntt, Kandolf, and CVB-RD strains of CVB3 with an equal volume containing $150 \mu \mathrm{g} / \mathrm{ml}$ of DAF-Fc (final concentration of $75 \mu \mathrm{g} / \mathrm{ml}$ ) resulted in varying degrees of infection inhibition. The RD cell-propagated CVB-RD strain was the most sensitive, whereas the HeLa cell-passaged Kandolf strain was not inhibited at all.

infection (coDAF), and 3 days after infection (postDAF). Results in treatment groups were compared with mice receiving virus only (Virus) or $100 \mu$ l of sterile PBS (Sham). There was no difference in mortality between virus-infected groups in the presence or absence of DAF-Fc treatment. Each infected group (including preDAF, coDAF, postDAF, and virus alone) had one death, and the overall mortality was $12.5 \%$ (4 of 32 mice). All mice were killed 7 days after infection.

Hematoxylin and eosin (H\&E) staining of infected hearts revealed that DAF-Fc administration before and during CVB3 infection caused significantly attenuated myocardial pathology as compared with DAF-Fc treatment after infection or virus alone (Fig. 4). In these heart sections, myocarditic lesional area was measured as total affected myocardium and myocyte cell death ranged from cytopathic vacuolization to coagulative necrosis. Dystrophic calcification was present in necrotic zones as determined by the presence of typical morphologic granular and diffuse cellular calcification, reflecting cell death in myocytes that have lost the ability to maintain normal calcium homeostasis. We have previously shown that inflammation, present in the perivascular space and focally within the myocardium, is primarily mononuclear in murine models, composed mainly of $T$ cells and also of macrophages and natural killer cells (Chow et al, 1988). Semiquantitative grades of these tissues revealed significant reduction in lesional area, cell necrosis, calcification, and inflammation in hearts in preDAF or coDAF, but not postDAF, mice as compared with virus alone (Fig. 5). There was a general absence of fibrosis in these short-term infections, as determined by Masson's trichrome stain (not shown), consistent with previous observations (Chow et al, 1988). Similarly, in situ hybridization for viral positive strand revealed decreased viral genomic RNA in the preDAF and coDAF groups as compared with hearts from the postDAF and virus alone groups (Fig. 4). Serial sections of H\&E and those submitted to in situ 
H\&E

\section{Sham}

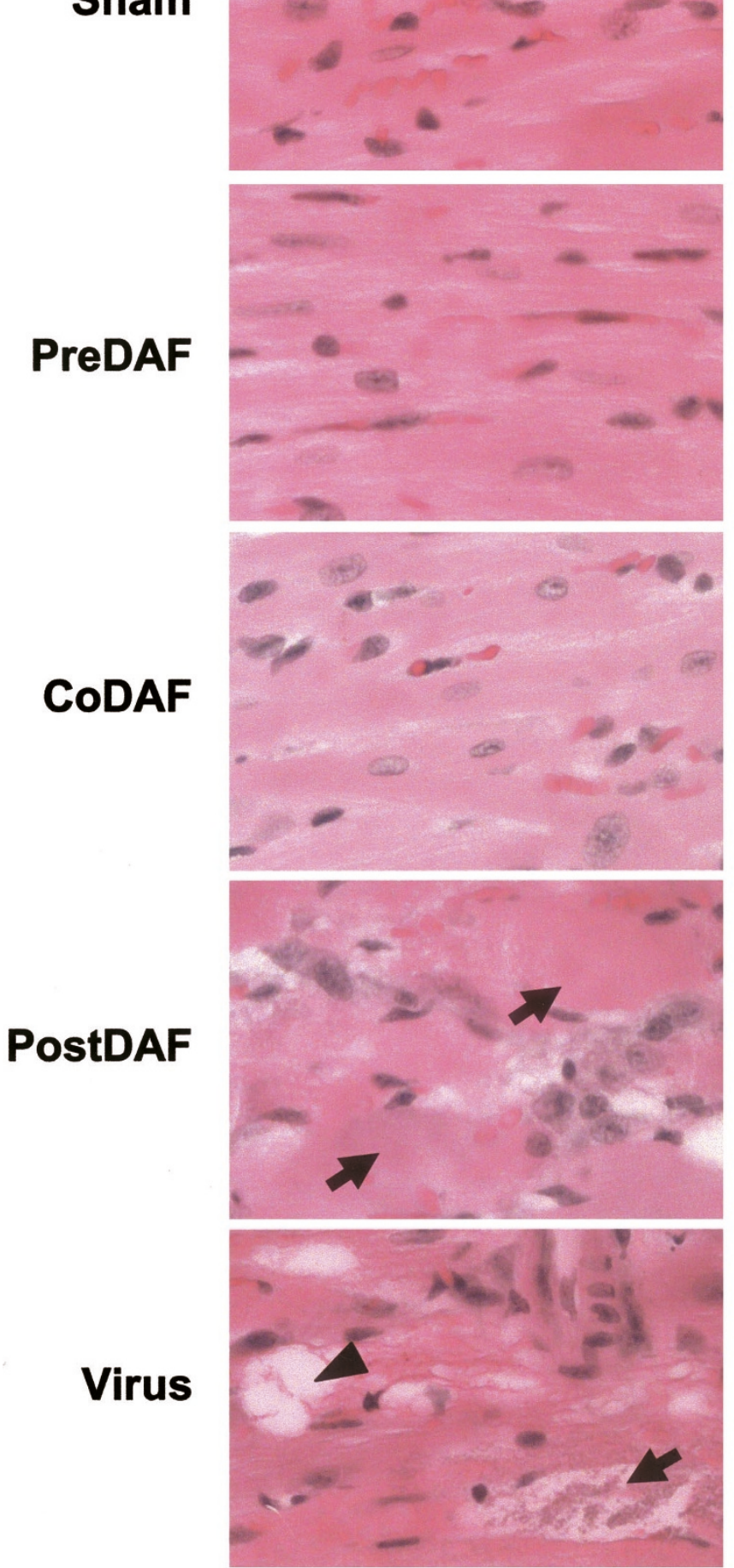

\section{In Situ Hybridization}
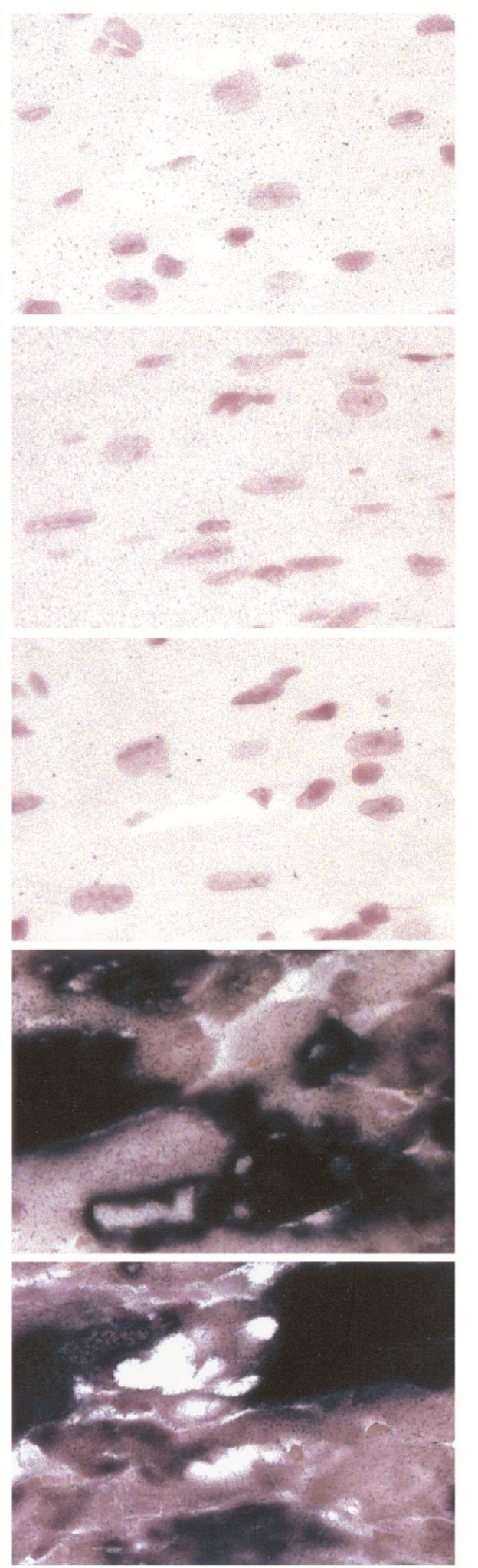

\section{Figure 4.}

DAF-Fc-attenuated myocarditic pathogenesis and virus replication. Adolescent A/J mice were infected with $10^{5}$ plaque-forming units of CVB3 (Gauntt) in the presence or absence of DAF-Fc 3 days before, during, and 3 days after infection or sham-infected (8 mice/group) and killed on Day 7 . Heart sections are stained with hematoxylin and eosin and in situ hybridization for viral positive-strand RNA $(\times 40)$. Cell death is prominent in postDAF tissue (arrow), which closely resembles virus group tissue containing intracellular calcification (arrowhead) and vacuolization (arrow) localized to areas of in situ positivity. PreDAF and coDAF tissue is similar to sham tissue with preservation of myocardial integrity and few areas of in situ positivity. 

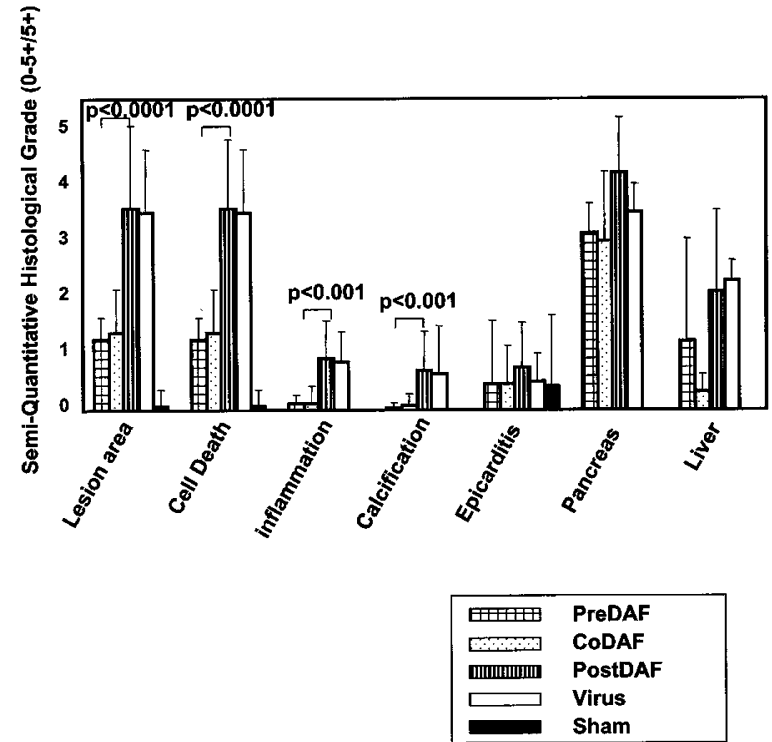

Figure 5.

DAF-Fc protects again myocarditis. Heart tissues were semiquantitatively graded based on lesion area, cell necrosis, calcification, and epicarditis (mean $\pm \mathrm{SD}, n=7$ or 8). Pancreas and liver were also graded for pathology. Significant differences were observed for the preDAF and coDAF groups as compared with the postDAF and virus only groups for myocardial lesion area, cell death, inflammation, and calcification (ANOVA). Epicarditis is normally observed in $\mathrm{A} / \mathrm{J}$ mice and is considered unrelated to virus infection. The exocrine pancreas is particularly susceptible to infection and exhibits extensive pathology regardless of HDAF-Fc treatment. Sham tissues were negative for all parameters.

hybridization showed colocalization of myocardial cell death and virus RNA, showing that viral replication contributes to observed myocardial pathogenesis (Fig. 4) (McManus et al, 1993). The exocrine pancreas is exceptionally sensitive to coxsackievirus infection and, in this study, showed extensive cell death and the presence of viral RNA in all virus groups regardless of treatment. Highly variable yet reduced liver pathology was observed with early treatment with DAF-Fc. The liver was in situ negative, suggesting that virus replication may occur either before our 7 day timepoint or not all (not shown). It has previously been shown that different strains of mice vary widely in hepatic involvement despite intense myocarditis (Grun et al, 1988). Quantitative image analysis of in situ hybridization of heart sections showed that the preDAF and coDAF treatment groups, but not the postDAF group, had significant decreases in viral RNA as compared with virus alone (Fig. 6). Plaque assay of heart tissues confirmed that DAF-Fc appreciably reduced infective virus particles for all treatment groups (Fig. 7).

\section{Assessing the Effects of Fc In Vivo}

To determine the immunomodulatory effects of the Fc region of DAF-Fc, we used two controls administered at Day 0 in a subset of mice and compared these results to virus alone. Fc directly cleaved from DAF-Fc $(n=4)$, commercially available, purified whole human $\operatorname{lgG}(n=4)$, or PBS $(n=2)$ was administered to mice

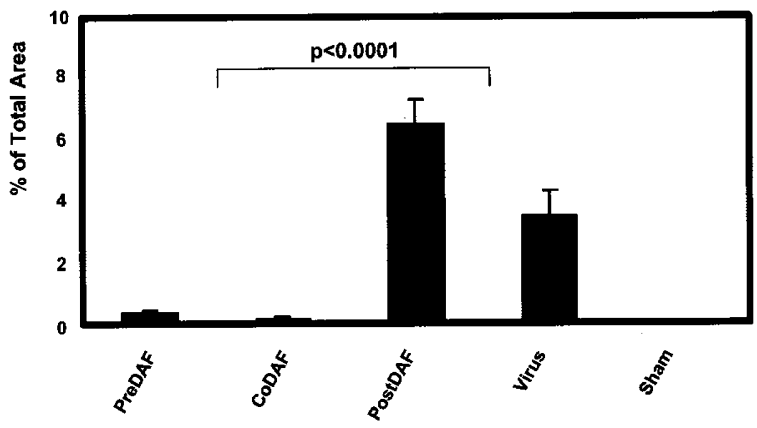

Figure 6.

DAF-Fc reduces virus replication. Total area of positive CVB3 in situ hybridization staining was quantified using ImageProPlus and expressed as a percentage of total cross-sectional area of the heart (mean $\pm \mathrm{SD}, n=7$ or 8 ). Total area of positive staining was quantified and standardized using ImageProPlus and expressed as a percentage of total cross-sectional area of the heart. PreDAF and DAF treatment showed significant attenuation of viral RNA as compared with postDAF and virus alone (ANOVA).

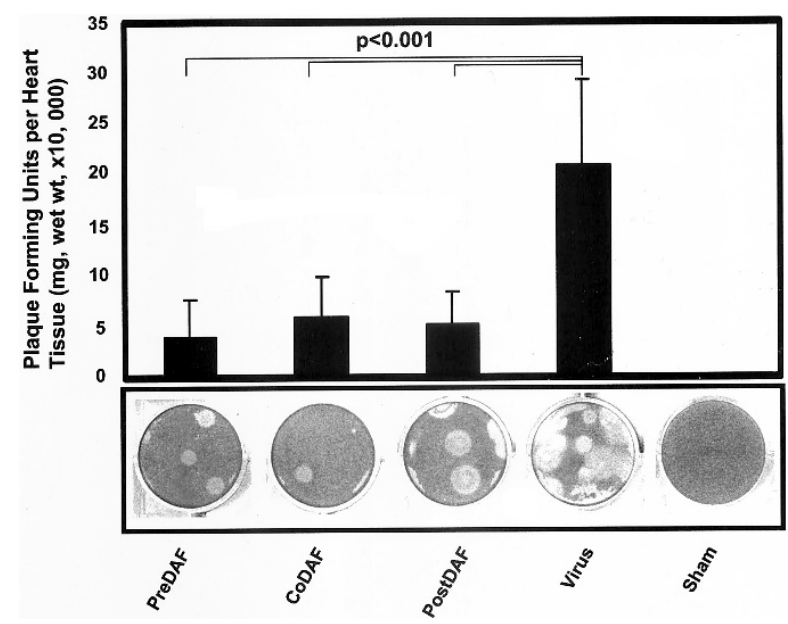

Figure 7.

DAF-Fc reduces infective virion. Cardiac tissue (apex) was homogenized and viral titers were assessed by plaque assay (mean $\pm \mathrm{SD}, n=7$ or 8 ). DAF-FC treatment at all time points with virus infection exhibited significantly reduced virus titers as compared with virus alone (ANOVA).

via intravenous injection on Day 0 . Mice were then infected with CVB3 by intraperitoneal inoculation as above and then killed for analysis at 7 days after infection. Semiquantitative histologic grades revealed decreased myocardial cell death and lesion area (Fig. 8A) but only $50 \%$ of that seen for whole DAF-Fc (Fig. 5); however, it is important to note that mice treated with Fc controls showed no reduction in inflammation, which contrasts with the effects of DAF-Fc. Image analysis for in situ hybridization staining of CVB3 RNA revealed a 3-fold decrease in positive-strand CVB3 RNA with either cleaved Fc or whole IgG controls (Fig. 8B), as compared with almost complete abrogation of CVB3 presence for mice treated with DAF-Fc (Fig. 6). Interestingly, there was a subtle decrease in infectious virus particles in heart homogenate with cleaved Fc as compared with the strong decrease observed in mice treated with whole IgG (Fig. 8C). 
A

B
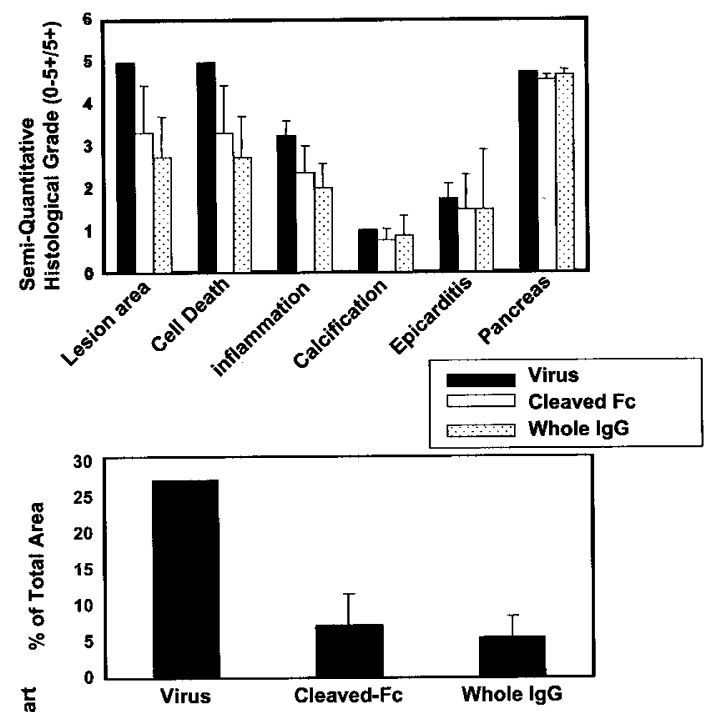

c

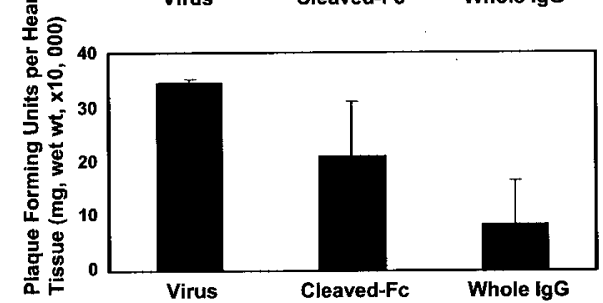

Figure 8.

Cleaved $\mathrm{Fc}$ and whole $\mathrm{IgG}$ reduce virus replication and myocardial injury. Mice were given either Fc protein cleaved from DAF-Fc, whole human IgG, or PBS (Day 0), infected with CVB3, and killed at Day 7. Heart tissue was submitted for histologic grading, quantitation of in situ hybridization for virus positive-strand RNA, and plaque assay to detect infectious virus particles. Cleaved Fc, and to a greater extent whole IgG, reduced myocarditic cell death and lesion area (A), virus replication $(B)$, and infectious virus particles $(C)$ in the heart.

\section{Discussion}

The ability of CVB3 to use DAF as a receptor has been well characterized and is best illustrated through the ability of preincubation with anti-DAF antibodies to block CVB3 infection of permissive cells (Bergelson et al, 1995, 1997; Martino et al, 1998, 2000; Shafren et al, 1997; Schmidtke et al, 2000). This is the first report to show that preincubation of soluble recombinant DAF with CVB3 virions blocked infection of permissive cells. A similar inhibition of in vitro CVB3 infection has also been shown by Martino et al (2000) using soluble recombinant CAR-fc. DAF-Fc was chosen over monomeric soluble DAF because of the enhanced ease of purification (Lo et al, 1998) and increased in vivo stability (Powers et al, 2001). Expression of proteins as recombinant dimeric Fc fusion proteins has been shown to increase the in vivo beta-phase serum half-life from 3.5 hours to 93 hours (Powers et al, 2001).

The ability of DAF-Fc to block CVB3 infection varied from strain to strain, similar to the variation reported for blocking infection with anti-DAF antibody (Bergelson et al, 1997). DAF-Fc strongly blocked infection by CVB-RD strain CVB3 and reduced infection by Gauntt strain CVB3 but had little effect on infection by Kandolf strain CVB3 to prevent cytopathic effect and cell death
(Fig. 3). The Gauntt and Kandolf strains have been propagated in HeLa cells (which expressed both CAR and DAF) with occasional passages in mice, whereas the CVB-RD strain has been propagated serially in rhabdomyosarcoma (RD) cells (which expressed DAF but not CAR). CVB3, which has been adapted to replicate in RD cells or human fibroblasts, has been previously shown to enhance CVB3 binding to DAF, and this has been attributed to the negligible expression of CAR on these cells (Bergelson et al, 1995; Pasch et al, 1999; Reagan et al, 1984; Schmidtke et al, 2000; Tracy et al, 1997). We found that neither the Gauntt and Kandolf strains would grow in RD cells (not shown). Furthermore, there is evidence to suggest that serial passage of CVB3 in RD cells can attenuate the CVB3-associated pathogenicity in mice (Fohlman et al, 1993; Landau et al, 1990). Because we sought to identify the ability of DAF-Fc to attenuate CVBassociated cardiomyopathy, we chose the highly cardiovirulent Gauntt strain for the in vivo studies, despite the fact that in vitro inhibition of CVB3 infection by DAF-Fc was only 10 -fold compared with the $10^{4}$-fold achieved with the CVB-RD strain (Fig. 3). DAF-FC would not compete for mouse DAF-binding in vivo because we have previously shown that CVB3 cannot use mouse DAF as a receptor (Spiller et al, 2000); therefore, infection of mouse cells would be entirely mediated by mouse CAR (Bergelson et al, 1998).

The primary function of DAF is not as a virus receptor: DAF is a potent regulator of complement activation in all mammals including humans and mice (Harris et al, 2000). Complement, part of the innate immune system, is an amplification cascade that is activated by invading pathogens. Complement enhances the recruitment of the cellular immune system and enhances the production of antibodies as well as directly destroying pathogens or pathogen-infected cells through formation of lytic pores (Barrington et al, 2001; Walport, 2001). We found that monomeric DAF and DAF-Fc were both capable of regulating erythrocyte lysis by human serum in vitro (Fig. 2), confirming that expression of DAF as an $\mathrm{Fc}$-fusion protein does not remove the complement-regulating ability. Furthermore, we have previously reported that human DAF-Fc is capable of regulating the alternative complement activation pathway of mouse serum in vitro (Harris et al, 2000), and the amounts of DAF-Fc we used in vivo were considerably greater than those used to inhibit mouse complement in vitro. Our previous studies investigating the role of complement in the pathogenicity of CVB3 in mice found that virus protein retention in splenic germinal centers was mediated by the mouse alternative complement pathway, and the CVB3 viral RNA localized to the secondary follicle germinal centers is thought to be primarily associated with B cells (Anderson et al, 1996). However, in the present study, there was no difference in splenic localization of CVB3 between treatment groups as compared with virus alone (not shown), which may suggest that the decreased pathogenicity seen with DAF-Fc treatment may not be a result of the inhibition of complement activation. 
In our well-established murine model of viral myocarditis, we administered DAF-Fc before, concurrent with, and after CVB3 infection to assess the efficacy in attenuating virus infection and myocardial injury. We have previously reported peak virus replication to occur at 3 days after infection and early inflammation at 5 to 7 days after infection in this model (McManus et al, 1993). Therefore, all mice were killed on Day 7 to assess treatment efficacy at peak myocardial inflammation and damage. There was no difference in rates or times of spontaneous mortality for treatment groups as compared with virus alone, suggesting that DAF-Fc does not prevent early mortality. We have previously shown that Gauntt strain CVB3 infection of $\mathrm{A} / \mathrm{J}$ mice resulted in panmural myocarditic lesions, severe dystrophic calcification, and high virus titers (Chow et al, 1991). Histologically, we assessed features of myocardial pathogenesis that included cell death, calcification, inflammatory infiltration, and the extent of area involvement (Fig. 5). Treatment either before or concurrent with virus infection significantly attenuated myocardial pathology. We have previously found that epicarditis is inherent to the $\mathrm{A} / \mathrm{J}$ mice and its severity is unrelated to virus infection (Chow et al, 1991), and no difference was observed between the treatment groups and virus alone in this study. Delayed administration of DAF treatment resulted in a negligible protective effect for cardiomyopathy. This suggests that the direct myocardial damage by the virus or the complement-mediated damage occurred very early in virus infection, indicating that DAF is only effective as a therapeutic agent if administered before the peak viremic phase.

Because DAF-Fc was capable both of regulating complement and of inhibiting virus infection directly in vitro, the exact mechanism by which DAF-Fc decreases myocardial damage is unclear. Pretreatment and cotreatment of animals with DAF-Fc was found to be far more effective at reducing pathology compared with posttreatment. There are a few possible explanations for this: (1) DAF-Fc already present in the circulation in pretreatment and cotreatment groups would neutralize or reduce the infectious viral load before direct infection of the myocardium and reduce spread of secondary virus infection at primary foci of infection. (2) CVB infection activates complement and DAF-Fc is capable of regulating mouse complement activation, limiting complement-mediated damage to CVB-infected cells and surrounding healthy tissue. (3) DAF-Fc inhibition of complement activation decreases the production of bioactive complement fragments $\mathrm{C} 3 \mathrm{a}$ and $\mathrm{C} 5 \mathrm{a}$, thereby reducing the infiltrating cellular component of inflammation. In truth, the effectiveness of DAF-Fc as a treatment for CVB-mediated myocarditis is probably a consequence of all three of these effects; the decreased myocardial virus titers observed with DAF-Fc treatment indicate that the effect cannot be solely explained by complement inhibition. We are currently designing Fc-fusion proteins that inhibit complement but do not bind to CVB3 and that bind to virus but do not inhibit complement to address the relative contributions of each in myocardial dam- age. However, it may transpire that DAF-Fc is a superior therapeutic agent in further experiments because of its broad-spectrum abilities.

The primary reason for using DAF in the form of an Fc fusion protein was to minimize the in vivo clearance so that a single intravenous injection would be sufficient for the course of the experiment. Recently, Harris et al (2002) showed that soluble DAF was rapidly cleared after intravenous injection of rats (half-life of 20 minutes); however, $28 \%$ of the functional activity for DAF-Fc still remained at 48 hours. Intravenously administered immunoglobulins have been found to have a beneficial effect in both clinical and experimental models of myocarditis (Drucker et al, 1994; Takada et al, 1995; Tedeschi et al, 2002), and there are also specific reports of intravenous polyvalent human IgG being used to treat human patients with CVB-related pathology (Geller and Condie, 1995; Isacsohn et al, 1994; Valduss et al, 1993). We have confirmed that whole human IgG reduces pathology, in our mouse model (Fig. 8). However, much of this is probably a result of the specific anti-CVB3 reactivity that exists in the normal IgG pool used as a source of the reagent, which is emphasized by the ability of whole human IgG to reduce virus recovered from myocardium compared with the minimal effect of cleaved Fc (Fig. 8C). The decrease in myocarditic grades and the virus RNA presence in the mice treated with cleaved Fc were unexpected, even though they were still $50 \%$ less active than DAF-Fc; however, this may be related to the recent findings that CAR, the only CVB receptor identified in mice, has been found to bind human IgG and IgM (Carson and Chapman, 2001). Therefore, the Fc portion may act to inhibit virus-receptor interactions by binding to CAR, while the DAF portion may inhibit the receptor-virus interactions by binding the virus.

In most instances, CVB3 infection in the general population is insidious and often subclinical. Also, in a clinical setting, patients often present during the acute inflammatory stage of viral myocarditis, wherein a treatment such as DAF-Fc would not be able to prevent the initial infectious process. However, subsets of the population, such as neonatal patients, experience a pernicious, rapid-onset, lifethreatening infection (Batra and Lewis, 2001). Kaplan et al (1983) documented 77 enterovirus infections in neonatal patients during a 9-year period, of which 8 were fatal because of overwhelming virus infection. In addition, outbreaks resulting from coxsackievirus infections in nurseries are of particular concern. In such situations wherein diagnosis is very rapid, antiviral agents such as DAF-Fc may be efficacious in attenuating acute coxsackievirus infections, but further studies are needed to characterize this novel potential therapeutic agent. DAF-Fc treatment may also prevent chronic infection that will lead to cardiac dilatation, and we are also designing experiments to study the longer term effects of DAF-Fc therapy in CVB3 infection. 


\section{Materials and Methods}

\section{Generation and Purification of DAF-Fc Fusion Protein}

The DAF-Fc fusion protein was created as described previously (Harris et al, 2000). DNA encoding the first four SCRs was amplified by PCR from full-length human DAF cDNA, which was a generous gift of Dr. I. Anegon (INSERM U437, Nantes, France). PCR products were ligated into the expression vector Signal plgplus (R\&D Systems, Minneapolis, Minnesota), according to the manufacturer's instructions, ensuring that it was in-frame with DNA encoding the hinge and Fc regions of human IgG1. Expression resulted in soluble forms of DAF consisting of the four SCRs linked to the $\mathrm{Fc}$ domain of human IgG1 (DAF-immunoglobulin).

To obtain high levels of secretion, DNA encoding the DAF-Fc fusion protein was subcloned into the expression vector, pDR $2 \delta \mathrm{EF} 1 \alpha$. Sequencing confirmed that no errors had been introduced when PCR was performed. Chinese hamster ovary cells were transfected with plasmid, as described previously (Harris et al, 1999), stable cell lines were generated, and culture supernatant was harvested. DAFimmunoglobulin constructs were purified from the supernatant by protein A affinity chromatography according to the manufacturer's instructions (Prosep A; Bioprocessing Ltd.). Because of the nature of the IgG1 Fc portion of the fusion protein, the product is dimeric and has an $M_{r}=150 \mathrm{kDa}$. Monomeric human DAF was released by a 20-minute incubation with agaroseimmobilized papain (Sigma-Aldrich), and the cleaved Fc portion and uncleaved DAF-Fc were removed by protein A affinity chromatography. Purity was assessed by SDS-PAGE, and concentration was determined by sandwich ELISA using a mouse monoclonal capture antibody (MBC1; a generous gift from Dr. C. L. Harris, University of Wales College of Medicine, Cardiff, United Kingdom) and a rabbit polyclonal antiDAF detection antibody.

\section{In Vitro Coxsackie B Virus Neutralization by DAF-Fc}

Stock CVB3 was generously provided by Drs. Charles Gauntt (University of Texas Health Sciences Center, San Antonio, Texas), Reinhard Kandolf (University of Tubingen, Germany), and David Evans (CVB-RD; University of Glasgow, United Kingdom). Gauntt and Kandolf CVB3 was propagated in HeLa cells, whereas CVB-RD CVB3 was propagated in RD cells. All viruses were stored at $-80^{\circ} \mathrm{C}$, and titers were routinely determined at the beginning of all experiments. Because the CVB-RD strain has been tissue culture adapted to $\mathrm{RD}$ cells, it does not grow to high titers in HeLa cells. HeLa cells (cervical epithelia) and RD cells were purchased from the European Collection of Cell Cultures (Salisbury, United Kingdom), and the IB-RS-2 pig kidney cell line was purchased from the American Type Culture Collection (Manassas, Virginia). All cells were grown in DMEM containing 10\% FCS and supplemented with glutamine (2 mM), sodium pyruvate (1 $\mathrm{mm})$, penicillin $(100 \mathrm{U} / \mathrm{ml})$, and streptomycin $(100 \mu \mathrm{g} /$ $\mathrm{ml}$ ). A 10-fold dilution series was made for each of the three virus stocks, and a $50-\mu$ l aliquot of each was incubated with a $50-\mu$ l aliquot containing $150 \mu \mathrm{g} / \mathrm{ml}$ DAF-Fc or serum-free DMEM as a control. Preliminary experiments determined that maximum inhibition of CVB3 infection was attained with preincubation with a final concentration of $75 \mu \mathrm{g} / \mathrm{ml}$ of DAF-Fc with virus for 30 minutes on ice (not shown). After allowing the virus and DAF-Fc (or control) to incubate on ice for 30 minutes, the entire mixture was overlaid onto a 96-well plate containing $50 \%$ confluent IB-RS-2 cells and the infection was allowed to proceed for 72 hours. IBRS-2 cells were chosen because Gauntt and Kandolf strains do not cause cytopathic effects in RD cells and the CVB-RD strain grows poorly in HeLa cells (not shown); however, all three strains cause a similar cytopathic effect in IB-RS-2 cells. The remaining viable cells were visualized by staining with $0.1 \%$ cresyl violet and $0.1 \%$ formaldehyde in PBS. Successful blockade of infection was observed as a viable blue cell layer at 3 days after infection. All experiments were repeated several times on different days to assure reproducibility of results.

\section{Complement Inhibition by DAF-Fc}

A series of 2-fold dilutions of DAF-Fc or monomeric DAF were made in veronal-buffered saline, and $50 \mu \mathrm{l}$ was added to $50 \mu \mathrm{l}$ of human serum (diluted 1/80). Fifty microliters of $1.0 \%$ washed sheep erythrocytes, preincubated with rabbit polyclonal sensitizing antibody, was then added; the amount of lysis, after 1-hour incubation at $37^{\circ} \mathrm{C}$, was determined by release of hemoglobin (measured at $415 \mathrm{~nm}$ ). All assays were performed in triplicate, and successful blocking was calculated as the percent reduction in hemoglobin release at the end of the assay.

\section{Virus Infection In Vivo}

Forty adolescent $\mathrm{A} / \mathrm{J}$ mice were randomly placed into five groups of eight mice and either infected intraperitoneally with $10^{5} \mathrm{PFU}$ of Gauntt strain CVB3 previously described as CVB3-CG (Chow et al, 1991) (four groups) or sham infected. Mice were administered DAF-Fc $(10 \mathrm{mg} / \mathrm{kg})$ intravenously 3 days before infection, concurrent with infection, and 3 days after infection or given virus alone. All mice were killed on Day 7. Heart, spleen, pancreas, kidney, and liver were harvested for subsequent analysis.

A subset of adolescent $\mathrm{A} / \mathrm{J}$ mice were intravenously administered either Fc cleaved from DAF-Fc $(n=4)$, purified whole human IgG $(n=4)$ purchased from Sigma-Aldrich, or PBS $(n=2)$ and infected with CVB3 as above. All mice were killed on Day 7 , and tissues were collected as above.

\section{Histology, In Situ Hybridization, and Semiquantitative Histologic Analysis}

Mid ventricular portions of heart specimens, as well as liver, pancreas, kidney, and spleen, were formalin fixed and embedded in paraffin; $4-\mu \mathrm{m}$ sections were 
cut and stained with either H\&E or Masson's Trichrome stain. Sections were also submitted for viral positive-strand and negative-strand RNA using in situ hybridization as previously described (Anderson et al, 1996). H\&E sections were graded for the extent of myocarditis based on the intensity and character of injury and inflammatory infiltration as previously described (Chow et al, 1991). Myocardial lesion area, cell death, calcification, and epicarditis were graded without knowledge of the experimental group (BMM). Grades were based on the following scale: 0, no or questionable presence; 1, limited focal distribution; 2-3, intermediate severity; and 4-5, coalescent and extensive foci over the entirety of the transversely sectioned ventricular tissue. For a more quantitative evaluation of the infection, ImageProPlus software was used to quantify the percent of total crosssectional area containing CVB3 RNA. A segmentation file that recognized positive in situ hybridization staining on the basis of hue, saturation, and intensity was developed. The total area of staining was measured and expressed as a percent of total cross-sectional area. All statistical analyses were performed using paired Student's $t$ test and ANOVA.

\section{Plaque Assay}

The amount of CVB3 in cell supernatant or cytoplasmic extracts was determined on monolayers of HeLa cells by the agar overlay plaque assay method as previously described (Anderson et al, 1996). Briefly, cardiac tissue (apex) was homogenized and solubilized. Samples were serially diluted 10 -fold and overlaid on $90 \%$ to $95 \%$ confluent monolayers of HeLa cells in 6-well plates (Corning Costar) and incubated for 1 hour $\left(5 \% \mathrm{CO}_{2}, 37^{\circ} \mathrm{C}\right)$. Media containing nonbound virus was removed, and warm complete MEM containing $0.75 \%$ agar was overlaid in each well. The plates were incubated for 36 to 48 hours $\left(5 \% \mathrm{CO}_{2}\right.$, $\left.37^{\circ} \mathrm{C}\right)$, fixed with Carnoy's fixative $(95 \% \mathrm{EtOH}$, acetic acid [3:1]), and stained with $1 \%$ crystal violet. The plaques were counted, and the viral concentration was calculated for seven to eight mice per group as PFU per milliliter of culture supernatant.

\section{Acknowledgement}

We thank Dr. Claire L. Harris (University of Wales College of Medicine) for her work on complement inhibitor-Fc fusion protein development and refinement. Without her, none of these experiments would have been possible.

\section{References}

Anderson DR, Wilson JE, Carthy CM, Yang D, Kandolf R, and McManus BM (1996). Direct interactions of coxsackievirus B3 with immune cells in the splenic compartment of mice susceptible or resistant to myocarditis. J Virol 70:4632-4645.

Barrington R, Zhang M, Fischer M, and Carroll MC (2001). The role of complement in inflammation and adaptive immunity. Immunol Rev 180:5-15.
Batra AS and Lewis AB (2001). Acute myocarditis. Curr Opin Pediatr 13:234-239.

Bauer S, Gottesman G, Sirota L, Litmanovitz I, Ashkenazi S, and Levi I (2002). Severe Coxsackie virus B infection in preterm newborns treated with pleconaril. Eur $\mathrm{J}$ Pediatr 161:491-493.

Bergelson JM, Krithivas A, Celi L, Droguett G, Horwitz MS, Wickham T, Crowell RL, and Finberg RW (1998). The murine CAR homolog is a receptor for coxsackie B viruses and adenoviruses. J Virol 72:415-419.

Bergelson JM, Modlin JF, Wieland-Alter W, Cunningham JA, Crowell RL, and Finberg RW (1997). Clinical coxsackievirus B isolates differ from laboratory strains in their interaction with two cell surface receptors. J Infect Dis 175:697-700.

Bergelson JM, Mohanty JG, Crowell RL, St. John NF, Lublin DM, and Finberg RW (1995). Coxsackievirus B3 adapted to growth in RD cells binds to decay-accelerating factor (CD55). J Virol 69:1903-1906.

Carson SD and Chapman NM (2001). Coxsackievirus and adenovirus receptor (CAR) binds immunoglobulins. Biochemistry 40:14324-14329.

Carthy CM, Granville DJ, Watson KA, Anderson DR, Wilson JE, Yang D, Hunt DW, and McManus BM (1998). Caspase activation and specific cleavage of substrates after coxsackievirus B3-induced cytopathic effect in HeLa cells. J Virol 72:7669-7675.

Chow LH, Beisel KW, and McManus BM (1992). Enteroviral infection of mice with severe combined immunodeficiency: Evidence for direct viral pathogenesis of myocardial injury. Lab Invest 66:24-31.

Chow LH, Gauntt CJ, and McManus BM (1991). Differential effects of myocarditic variants of coxsackievirus B3 in inbred mice: A pathologic characterization of heart tissue damage. Lab Invest 64:55-64.

Chow LH, Gauntt CJ, Ye YL, and McManus BM (1988). Early cellular infiltrates in coxsackievirus B3 murine myocarditis. In: Schultheib HP, editor. New concepts in viral heart disease. Munich: Springer-Verlag, 205-214.

Cohen CJ, Shieh JT, Pickles RJ, Okegawa T, Hsieh JT, and Bergelson JM (2001). The coxsackievirus and adenovirus receptor is a transmembrane component of the tight junction. Proc Natl Acad Sci USA 98:15191-15196.

Drucker NA, Colan SD, Lewis AB, Beiser AS, Wessel DL, Takahashi M, Baker AL, Perez-Atayde AR, and Newburger JW (1994). Gamma-globulin treatment of acute myocarditis in the pediatric population. Circulation 89:252-257.

Fohlman J, Pauksen K, Morein B, Bjare U, llback NG, and Friman $G$ (1993). High yield production of an inactivated coxsackie B3 adjuvant vaccine with protective effect against experimental myocarditis. Scand J Infect Dis Suppl 88:103108.

Geller TJ and Condie D (1995). A case of protracted coxsackie virus meningoencephalitis in a marginally immunodeficient child treated successfully with intravenous immunoglobulin. J Neurol Sci 129:131-133.

Grun JB, Schultz M, Finkelstein SD, Crowell RL, and Landau BJ (1988). Pathogenesis of acute myocardial necrosis in inbred mice infected with coxsackievirus B3. Microb Pathog 4:417-430. 
Harris CL, Rushmere NK, and Morgan BP (1999). Molecular and functional analysis of mouse decay accelerating factor (CD55). Biochem J 341:821-829.

Harris CL, Spiller OB, and Morgan BP (2000). Human and rodent decay-accelerating factors (CD55) are not species restricted in their complement-inhibiting activities. Immunology 100:462-470.

Harris CL, Williams AS, Linton SM, and Morgan BP (2002). Coupling complement regulators to immunoglobulin domains generates effective anti-complement reagents with extended half-life in vivo. Clin Exp Immunol 129:198-207.

Isacsohn M, Eidelman AI, Kaplan M, Goren A, Rudensky B, Handsher R, and Barak Y (1994). Neonatal coxsackievirus group $B$ infections: Experience of a single department of neonatology. Isr J Med Sci 30:371-374.

Ito M, Kodama M, Masuko M, Yamaura M, Fuse K, Uesugi Y, Hirono S, Okura Y, Kato K, Hotta Y, Honda T, Kuwano R, and Aizawa $Y$ (2000). Expression of coxsackievirus and adenovirus receptor in hearts of rats with experimental autoimmune myocarditis. Circ Res 86:275-280.

Kaplan MH, Klein SW, McPhee J, and Harper RG (1983). Group B coxsackievirus infections in infants younger than three months of age: A serious childhood illness. Rev Infect Dis 5:1019-1032.

Krah DL and Crowell RL (1982). A solid-phase assay of solubilized HeLa cell membrane receptors for binding group B coxsackieviruses and polioviruses. Virology 118:148-156.

Kunin CM (1964). Cellular susceptibility to enteroviruses. Bact Rev 28:382-390.

Landau BJ, Whittier PS, Finkelstein SD, Alstein B, Grun JB, Schultz M, and Crowell RL (1990). Induction of heterotypic virus resistance in adult inbred mice immunized with a variant of Coxsackievirus B3. Microb Pathog 8:289-298.

Liu P, Aitken K, Kong YY, Opavsky MA, Martino T, Dawood F, Wen WH, Kozieradzki I, Bachmaier K, Straus D, Mak TW, and Penninger JM (2000). The tyrosine kinase p56lck is essential in coxsackievirus B3-mediated heart disease. Nat Med 6:429-434.

Liu PP and Opavsky MA (2000). Viral myocarditis: Receptors that bridge the cardiovascular with the immune system? Circ Res 86:253-254.

Lo KM, Sudo Y, Chen J, Li Y, Lan Y, Kong SM, Chen L, An Q, and Gillies SD (1998). High level expression and secretion of Fc-X fusion proteins in mammalian cells. Protein Eng 11:495500.

Maisch B, Hufnagel G, Schonian U, and Hengstenberg C (1995). The European Study of Epidemiology and Treatment of Cardiac Inflammatory Disease (ESETCID). Eur Heart J 16 (Suppl O):173-175.

Martino TA, Petric M, Brown M, Aitken K, Gauntt CJ, Richardson CD, Chow LH, and Liu PP (1998). Cardiovirulent coxsackieviruses and the decay-accelerating factor (CD55) receptor. Virology 244:302-314.

Martino TA, Petric M, Weingartl H, Bergelson JM, Opavsky MA, Richardson CD, Modlin JF, Finberg RW, Kain KC, Willis N, Gauntt CJ, and Liu PP (2000). The coxsackie-adenovirus receptor (CAR) is used by reference strains and clinical isolates representing all six serotypes of coxsackievirus group $B$ and by swine vesicular disease virus. Virology 271:99-108.
Mason JW, JB OC, Herskowitz A, Rose NR, McManus BM, Billingham ME, and Moon TE (1995). A clinical trial of immunosuppressive therapy for myocarditis. The Myocarditis Treatment Trial Investigators. N Engl J Med 333:269-275.

McManus BM, Chow LH, Wilson JE, Anderson DR, Gulizia JM, Gauntt CJ, Klingel KE, Beisel KW, and Kandolf R (1993). Direct myocardial injury by enterovirus: A central role in the evolution of murine myocarditis. Clin Immunol Immunopathol 68:159-169.

Nicholson-Weller A, Burge J, Fearon DT, Weller PF, and Austen KF (1982). Isolation of a human erythrocyte membrane glycoprotein with decay-accelerating activity for C3 convertases of the complement system. J Immunol 129:184189.

Nicholson-Weller A and Wang CE (1994). Structure and function of decay accelerating factor CD55. J Lab Clin Med 123:485-491.

Pasch A, Kupper JH, Wolde A, Kandolf R, and Selinka HC (1999). Comparative analysis of virus-host cell interactions of haemagglutinating and non-haemagglutinating strains of coxsackievirus B3. J Gen Virol 80:3153-3158.

Powers DB, Amersdorfer P, Poul M, Nielsen UB, Shalaby MR, Adams GP, Weiner LM, and Marks JD (2001). Expression of single-chain Fv-Fc fusions in Pichia pastoris. J Immunol Methods 251:123-135.

Reagan KJ, Goldberg B, and Crowell RL (1984). Altered receptor specificity of coxsackievirus B3 after growth in rhabdomyosarcoma cells. J Virol 49:635-640.

Rossmann MG, Arnold E, Erickson JW, Frankenberger EA, Griffith JP, Hecht HJ, Johnson JE, Kamer G, Luo M, Mosser $A G$, et al (1985). Structure of a human common cold virus and functional relationship to other picornaviruses. Nature 317 : 145-153.

Rotbart HA and Webster AD (2001). Treatment of potentially life-threatening enterovirus infections with pleconaril. Clin Infect Dis 32:228-235.

Schmidtke M, Selinka HC, Heim A, Jahn B, Tonew M, Kandolf R, Stelzner A, and Zell R (2000). Attachment of coxsackievirus B3 variants to various cell lines: Mapping of phenotypic differences to capsid protein VP1. Virology 275: 77-88.

Shafren DR, Bates RC, Agrez MV, Herd RL, Burns GF, and Barry RD (1995). Coxsackieviruses B1, B3, and B5 use decay accelerating factor as a receptor for cell attachment. J Virol 69:3873-3877.

Shafren DR, Williams DT, and Barry RD (1997). A decayaccelerating factor-binding strain of coxsackievirus B3 requires the coxsackievirus-adenovirus receptor protein to mediate lytic infection of rhabdomyosarcoma cells. J Virol 71:9844-9848.

Shieh JT and Bergelson JM (2002). Interaction with decayaccelerating factor facilitates coxsackievirus B infection of polarized epithelial cells. J Virol 76:9474-9480.

Spiller OB, Goodfellow IG, Evans DJ, Almond JW, and Morgan BP (2000). Echoviruses and coxsackie B viruses that use human decay-accelerating factor (DAF) as a receptor do not bind the rodent analogues of DAF. J Infect Dis 181:340343. 
Spiller OB, Goodfellow IG, Evans DJ, Hinchliffe SJ, and Morgan BP (2002). Coxsackie B viruses that use human DAF as a receptor infect pig cells via pig CAR and do not use pig DAF. J Gen Virol 83:45-52.

Takada H, Kishimoto C, and Hiraoka Y (1995). Therapy with immunoglobulin suppresses myocarditis in a murine coxsackievirus B3 model: Antiviral and anti-inflammatory effects. Circulation 92:1604-1611.

Tedeschi A, Airaghi L, Giannini S, Ciceri L, and Massari FM (2002). High-dose intravenous immunoglobulin in the treatment of acute myocarditis: A case report and review of the literature. J Intern Med 251:169-173.

Tracy S, Chapman NM, and Mahy BWJ (1997). The coxsackie B viruses, 1st ed. Berlin: Springer-Verlag.
Valduss D, Murray DL, Karna P, Lapour K, and Dyke J (1993). Use of intravenous immunoglobulin in twin neonates with disseminated coxsackie B1 infection. Clin Pediatr (Phila) 32:561-563.

Walport MJ (2001). Complement: First of two parts. N Engl J Med 344:1058-1066.

Wang A, Cheung PK, Zhang $\mathrm{H}$, Carthy CM, Bohunek L, Wilson JE, McManus BM, and Yang D (2001). Specific inhibition of coxsackievirus B3 translation and replication by phosphorothioate antisense oligodeoxynucleotides. Antimicrob Agents Chemother 45:1043-1052.

Woodruff JF (1980). Viral myocarditis: A review. Am J Pathol 101:425-484. 